\title{
Integrated hydrological modelling of small- and medium-sized water storages with application to the upper Fengman Reservoir Basin of China
}

\author{
C. Zhang $^{1}$, Y. Peng ${ }^{1}$, J. Chu ${ }^{1}$, C. A. Shoemaker ${ }^{2}$, and A. Zhang ${ }^{1}$ \\ ${ }^{1}$ Institute of Water Resources and Flood Control, Dalian University of Technology, Dalian 116024, China \\ ${ }^{2}$ Department of Civil and Environmental Engineering, Cornell University, NY 14850, USA \\ Correspondence to: Y. Peng (pyongcuidi@163.com)
}

Received: 31 January 2012 - Published in Hydrol. Earth Syst. Sci. Discuss.: 28 March 2012

Revised: 2 August 2012 - Accepted: 11 October 2012 - Published: 6 November 2012

\begin{abstract}
Hydrological simulation in regions with a large number of water storages is difficult due to inaccurate water storage data. To address this issue, this paper presents an improved version of SWAT2005 (Soil and Water Assessment Tool, version 2005) using Landsat, a satellite-based dataset, an empirical storage classification method and some empirical relationships to estimate water storage and release from the various sizes of flow detention and regulation facilities. The SWAT2005 is enhanced by three features: (1) a realistic representation of the relationships between the surface area and volume of each type of water storages, ranging from small-sized flow detention ponds to medium- and large-sized reservoirs with the various flow regulation functions; (2) water balance and transport through a network combining both sequential and parallel streams and storage links; and (3) calibrations for both physical and human interference parameters. Through a real-world watershed case study, it is found that the improved SWAT2005 more accurately models small- and medium-sized storages than the original model in reproducing streamflows in the watershed. The improved SWAT2005 can be an effective tool to assess the impact of water storage on hydrologic processes, which has not been well addressed in the current modelling exercises.
\end{abstract}

\section{Introduction}

Water storages, ranging from small-sized flow detention ponds to medium- and large-sized reservoirs with the various flow regulation functions, are important management tools in the regulation and utilisation of water resources (Gross and Moglen, 2007; Lopez-Moreno et al., 2009) on one hand; on the other hand, they represent a major type of human interferences to hydrologic processes. Different approaches have been developed to account for the interferences in hydrological simulations. For example, water storage simulation models have been developed for small-sized river catchments (Jayatilaka et al., 2003; Saxton and Willey, 2004), which suggest that those models could be used as a useful tool for optimizing the usage of limited water resources in similar regions with a small amount of water storage. However, the impact of water storage on hydrological processes has not been well addressed in general, largely due to the fact that the popular watershed simulation models lack sufficient data and relationships to simulate the effects of water storages. To address this issue, this paper presents an improved version of SWAT2005 (Soil and Water Assessment Tool, version 2005) using Landsat, a satellite-based dataset, an empirical storage classification method and some empirical relationships to estimate water storage and release from the various sizes of flow detention and regulation facilities.

SWAT2005, one of the widely used distributed, physically based watershed simulation models (Neitsch et al., 2002a, b), uses hydrological responding units (HRUs) as the basic modelling units. Water storages can be explicitly modeled by appropriately parameterizing the corresponding HRUs (Payan et al., 2008). For example, to assess the impacts of water storages on streamflow in the Huai River Basin of China, Wang and Xia (2010) modeled 61 water storages in SWAT2000 by modifying the outflow calculation method 
for water storages. However, it turns out to be less feasible to use SWAT to account for a large number of water storages given the computational intensity for the distributed, physically based model with the small scales of measurements (i.e. small scale HRUs) (Sophocleous and Perkins, 2000) as well as the difficulty in obtaining the data for numerous water storages.

To reduce the computational requirement, Payan et al. (2008) proposed a way to account for man-made reservoirs in a lumped hydrological model. This model could not explicitly simulate the key processes in reservoirs (infiltration, evaporation, operation, and so on); instead, it used the observed volume variations to represent these processes. The model simplifications may not reasonably reflect various reservoir processes, particularly those in a large-scale, complex river catchment.

To represent several thousands of reservoirs located in the State of Ceará in semi-arid Northeast Brazil, Güntner et al. (2004) presented a simple deterministic water balance modelling scheme within a distributed model. The key component of the scheme was a cascade-type approach, within which the reservoirs were grouped into six classes according to storage capacity, each with different rules for flow routing. Water uses were considered for irrigation and livestock as well as domestic, industrial and tourist uses. The scheme assumed that the smaller-sized reservoirs were located upstream of larger-sized reservoirs and that the outflows from smaller-sized reservoirs were equally discharged into all of the larger-sized reservoir classes located downstream. To cope with data scarcity, particularly regarding water use and surface area, the scheme used empirical data of water use and an empirical formula to calculate surface area. Additionally, reservoir operation rules were not considered in the scheme. Refinements of the model should primarily focus on an improved definition of the basin area fractions contributing to individual reservoir classes by using more detailed data on topography and reservoir locations from remote sensing studies. Furthermore, a better knowledge of reservoir operation rules promises to significantly improve the performance of the model.

Therefore, the basin hydrologic cycle must be simulated accurately with all of the available information and reasonable modelling simplifications of the numerous water storages. Given the limitations of SWAT2005, the aim of this paper is to present an improved version of SWAT2005 to allow water storages in a large-scale river catchment to be simulated more accurately. Landsat, a satellite-based dataset, is used to obtain the surface area and volume of the various sizes of storages; an empirical storage classification method is employed to classify and aggregate some storages; and empirical relationships are developed to estimate water storage and release from the various sizes of flow detention and regulation facilities. The improved SWAT2005 is applied to the upper Fengman Reservoir Basin, which has a large number of small- and medium-sized water storages for irrigation, industrial, and domestic uses and the results are compared to those from the original SWAT2005.

\section{Methodology}

For a watershed with hundreds or thousands of water storages, it is not feasible to describe each water storage individually in a large-scale model. Thus, the approach of this study is not to exactly represent the behaviour of each water storage facility, but rather to model the water storage system on an aggregated level to allow water storages in a large-scale river catchment to be more reasonably simulated. Detailed design and operation information is known for all large- and medium-sized reservoirs. For small-sized reservoirs, only the geographic positions, drainage areas, emergency storage volumes, and regular storage volumes are available at the level of administrative units (municipalities). For ponds, only the total drainage areas and storage volumes can be obtained at the basin level. Therefore, water storages are grouped into five $\left(r_{\max }=5\right)$ classes depending on storage capacities $V_{\max }$ (Table 1).

SWAT is chosen for this study since it includes reservoir and pond modules, and the impact of storage can be assessed at a basin scale. SWAT has been widely used in a variety of investigations, such as hydrological simulation and assessment, non-point pollution, climate change impact, parameter sensitivity, and model calibration and uncertainty analysis (Borah and Bera, 2004; Arnold and Fohrer, 2005; Gassman et al., 2007).

The water balance used in the reservoir and pond modules of SWAT2005 is given as

$V=V_{\text {store }}+V_{\text {flow in }}-V_{\text {flow out }}+V_{\text {pcp }}-V_{\text {evap }}-V_{\text {seep }}$

where $V$ is the volume of water in reservoirs and ponds at the end of the day $\left(\mathrm{m}^{3}\right) ; V_{\text {stored }}$ is the volume of water stored in reservoirs and ponds at the beginning of the day $\left(\mathrm{m}^{3}\right) ; V_{\text {flow }}$ in is the volume of water entering reservoirs and ponds during the day $\left(\mathrm{m}^{3}\right) ; V_{\mathrm{pcp}}$ is the volume of precipitation falling on reservoirs and ponds during the day $\left(\mathrm{m}^{3}\right) ; V_{\text {evap }}$ is the volume of water removed from reservoirs and ponds by evaporation during the day $\left(\mathrm{m}^{3}\right)$; and $V_{\text {seep }}$ is the volume of water lost from reservoirs and ponds by seepage during the day $\left(\mathrm{m}^{3}\right)$.

The surface areas of water storages are needed to calculate the amount of precipitation falling on water storages and the amount of evaporation and seepage removed from water storages in SWAT. Surface area varies with the water volume of the water storages.

In SWAT2005, surface area is updated daily using the following equations

$$
\begin{aligned}
& \mathrm{SA}=\beta_{\mathrm{sa}} \cdot V^{\text {exsa }} \\
& \text { exsa }=\frac{\log _{10}\left(\mathrm{SA}_{\mathrm{em}}\right)-\log _{10}\left(\mathrm{SA}_{\mathrm{pr}}\right)}{\log _{10}\left(V_{\mathrm{em}}\right)-\log _{10}\left(V_{\mathrm{pr}}\right)}
\end{aligned}
$$


Table 1. Classification of water storages and the total drainage area and storage volume of each class in the Fengman Reservoir Basin.

\begin{tabular}{lrrrrrr}
\hline Class & 1 & 2 & 3 & 4 & 5 & Total \\
\hline Storage capacity $\left(10^{6} \mathrm{~m}^{3}\right)$ & $<0.1$ & $0.1 \sim 1.0$ & $1.0 \sim 10.0$ & $10.0 \sim 50.0$ & $>50.0$ & \\
Total drainage area $\left(\mathrm{km}^{2}\right)$ & 2252 & 1989 & 1902 & 730 & 548 & 7421 \\
Total storage volume $\left(10^{6} \mathrm{~m}^{3}\right)$ & 54.62 & 152.63 & 255.07 & 179.82 & 316.00 & 958.14 \\
\hline
\end{tabular}

$\beta_{\mathrm{sa}}=\left(\frac{\mathrm{SA}_{\mathrm{em}}}{V_{\mathrm{em}}}\right)^{\mathrm{exsa}}$

where SA is the surface area of the water storage (ha); $V$ is the volume of water in the water storage $\left(\mathrm{m}^{3}\right) ; \mathrm{SA}_{\mathrm{em}}$ is the surface area of the water storage when filled to the emergency spillway (ha); $\mathrm{SA}_{\mathrm{pr}}$ is the surface area of the water storage when filled to the regular spillway (ha); $V_{\mathrm{em}}$ is the volume of water held in the water storage when filled to the emergency spillway $\left(\mathrm{m}^{3}\right)$; and $V_{\mathrm{pr}}$ is the volume of water held in the water storage when filled to the regular spillway $\left(\mathrm{m}^{3}\right)$.

Güntner et al. (2004) calculated surface area as a function of the actual storage volume with

$A_{\mathrm{RL}}=c_{\mathrm{RL}} \cdot\left(V_{\mathrm{t}}\right)^{d_{\mathrm{RL}}}$

where $c_{\mathrm{RL}}$ and $d_{\mathrm{RL}}$ are reservoir-specific constants depending on its geometry.

Liebe et al. (2005) estimated storage volume as a function of surface area with

$V=0.00857 \cdot A_{\mathrm{Res}}^{1.4367}$

where $V$ is the volume of the water storage $\left(\mathrm{m}^{3}\right)$, and $A_{\mathrm{Res}}$ is its area $\left(\mathrm{m}^{2}\right)$.

Because the surface area of a water storage is related to its scope, storage and drainage area, the relationship between surface area and storage volume varies for the different water storage classes. It is not reasonable to use a definite relationship between surface area and storage volume to calculate the surface areas of different water storage classes, as done previously by Güntner et al. (2004), Liebe et al. (2005) and SWAT2005. In this paper, an approach for obtaining more precise relationships between the surface area and storage volume of different water storage classes is proposed.

Water balance and transport through a network combining both sequential and parallel streams and storage links is proposed, incorporating surface runoff and routing mechanisms based on the spatial topological relationships among water storages, the impoundment and release regulations of water storages with water uses.

The framework for the improved SWAT2005 is shown in Fig. 1 (the grey area shows the improvements). The reservoir module in SWAT can simulate water storages with detailed design and running information. The pond module in SWAT is an aggregate model of water bodies within any sub-basin.

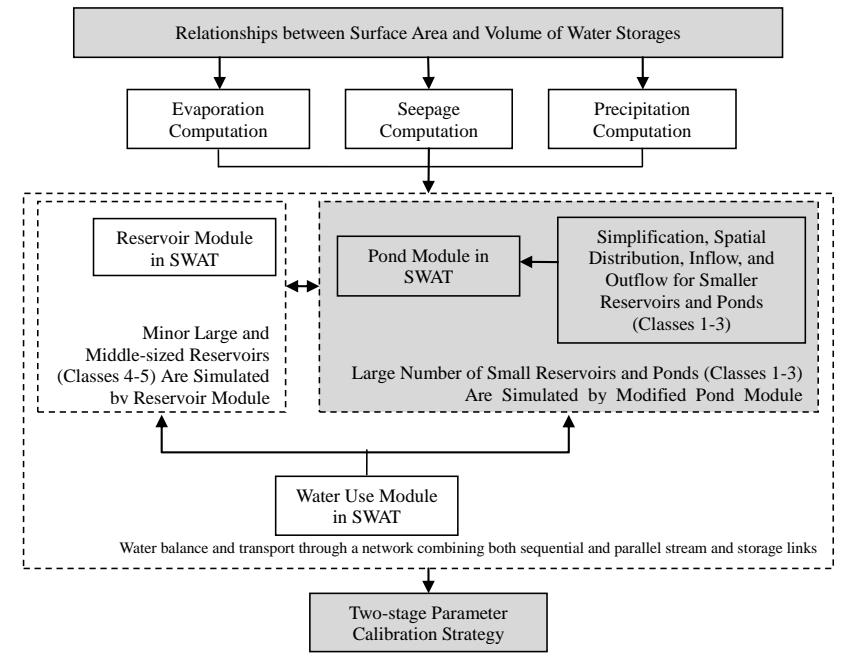

Fig. 1. Framework for the improved SWAT2005.

Therefore, in this paper, fewer large- and medium-sized reservoirs (classes 4 and 5) are added to SWAT2005 and simulated by the reservoir module of SWAT2005. Small-sized reservoirs and ponds (classes $1-3$ ) are simulated by the pond module, rather than the reservoir module of SWAT2005. The pond module of SWAT2005 treats all small-sized reservoirs and ponds within a sub-basin as an aggregated water storage, and surface runoff and routing processes between the storages are not considered. Although the pond module of SWAT2005 is a reasonable simplification, it can be improved in terms of relationships between surface area and volume of water storages, water balance and transport through a network combining both sequential and parallel streams and storage links, and calibration for both physical and human interference parameters.

Distributed, extensive water storage within a basin can significantly influence the basin hydrologic processes. The impacts must then be included in the parameterization and calibration of a hydrological simulation model. This study then presents a procedure to calibrate both physical and human interference parameters, e.g. parameters related to water storages and water consumption, which are major human interferences to hydrologic processes.

Additionally, water consumption is modeled as an item of water loss from the system. SWAT allows water to be withdrawn from a shallow aquifer, a deep aquifer, a river 
reach, or a water storage facility within any sub-basins. Water consumption varies from month to month, and the average daily volume of water consumption from a source needs to be specified by month. However, the associated and detailed water-use data, such as month water uses in different places and different years, are difficult to collect, and water-use data treatment based on parameter calibration is proposed in this paper.

\subsection{Relationships between surface area and volume of water storages}

\subsubsection{Surface area}

There have been several studies on obtaining the surface areas of small-sized reservoirs with satellite images (White, 1978; McFeeters, 1996; Frazier and Page, 2000). Optical (i.e. Landsat, Spot, Aster and ISS) or radar satellite systems (i.e. Envisat, ERS, and Radarsat) could be used to obtain the surface areas of small-sized reservoirs. Envisat ASAR (Cband radar) and Landsat TM/ETM+ data (multispectral imagery) now provide images at spatial resolutions of $30 \mathrm{~m}$ and $15 \mathrm{~m}$, respectively (Gardini et al., 1995). In India, the storage volumes of small-sized reservoirs were estimated with Landsat images (Mialhe et al., 2008). Envisat advanced synthetic aperture radar (ASAR) was used to obtain the surface areas of reservoirs (Liebe et al., 2009). At present, there are numerous approaches of extracting surface areas from remote sensing data, such as the single band threshold method (Ding, 2012; Du and Zhou, 1988), the difference of spectrum relationship method (Cao, 2006), the Normalized Difference Vegetation Index (NDVI) method (Wu et al., 2005; $\mathrm{Li}$ and $\mathrm{Wu}, 2008$ ) and the Normalized Difference Water Index (NDWI) method (McFeeters, 1996; Gao, 1995; Ouma and Tateishi, 2006). Water bodies have significant differences with beaches and shadows of mountains in reflectivity of bands TM2 and TM3, and lesser differences in bands TM4 and TM5, which make it easier to extract surface area with the improved spectrum relationship method. The spectrum is enhanced with $\frac{\mathrm{TM} 2+\mathrm{TM} 3}{\mathrm{TM} 4+\mathrm{TM} 5}$ in this method, and then a suitable threshold is chosen to extract surface area, as described below:

\section{$\frac{\mathrm{TM} 2+\mathrm{TM} 3}{\mathrm{TM} 4+\mathrm{TM} 5}>k 2$}

where $k 2$ is the threshold to extract surface area, $k 2 \in[1,2]$.

In this paper, the reservoir surface areas are extracted with Landsat TM/ETM+ data from 1986 to 2006 with the improved spectrum relationship method from the International Science Data Service Platform (http://datamirror.csdb.cn). Because Landsat TM/ETM+ provides high-resolution spatial data for every 16 days, the data items in flooding seasons in wet years are used to extract the surface areas of water storages within the basin. The extracted surface areas are assumed to go along with the regular storage volumes, which were collected from the Hydrological Administration of Jilin Province in China. Therefore, in this study, Landsat TM/ETM+ data from the flooding season (June-August) of a wet year (2005) are used to extract the surface areas of the water storages within the basin.

\subsubsection{Classifications of water storages and the relationship between surface area and regular storage volume for each water storage class}

Water storages with different slopes, storages, and drainage areas have different relationships between their surface area and storage volume. Therefore, the three elements (slope, storage, and drainage area) should be considered when developing such relationships for each water storage class. The ratio of drainage area to storage volume is set as an index to classify the water storages. For larger ratios, the storage volume increases more rapidly with the area and there is a higher probability of reaching the regular storage volume during wet periods. The water storage classification and acquiring the relationships between surface area and regular storage volume for each water storage class is conducted through a four-step approach.

Step 1: setting the initial classifications of water storages according to the value distributions of their classification indices (slope, drainage area/storage volume, and principle storage).

Step 2: calculating the mean characteristics of each water storage class, such as the mean storage volume, mean drainage area, and mean slope, and finding the medium-sized reservoir in the basin with similar mean characteristics. Because detailed design and running information are known for the medium-sized reservoirs, use the ratio of the storage volume of the medium-sized reservoir from 9 September 2005 to its regular storage volume as means to adjust the regular storage volume of the water storages within the water storage class.

Step 3: calculating the correlation coefficients between the surface area and regular storage volume of the water storages for each water storage class.

Step 4: adjusting the classification indices and repeating step 2 until the correlation coefficients could no longer be improved.

\subsection{Water balance and transport through a network combining both sequential and parallel streams and storage links}

In this paper, a sequential and parallel routing scheme is developed to approximately describe the upstream-downstream positions of different water storage classes within the subbasin and the runoff routing in the network. The major difference between the traditional cascade scheme and the 


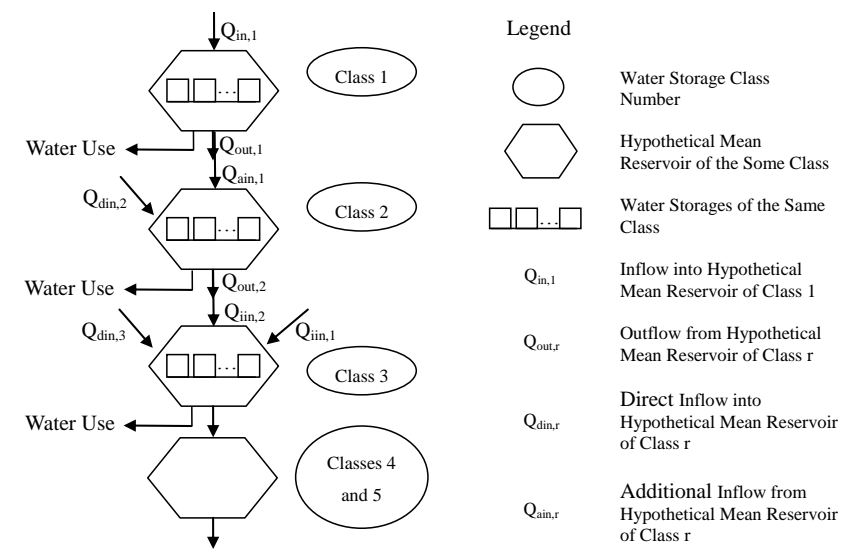

Fig. 2. Sequential and parallel routing scheme for water storages in the improved SWAT2005.

sequential and parallel routing scheme lies in the calculation of the inflow and outflow from water storages. More specifically, the latter divides the water storages into two simulation classes: one with large- and medium-sized reservoirs and the other with small-sized reservoirs and ponds. The simulation of these two classes with consideration of the variability of spatially distributed water uses during different years is described below.

\subsubsection{Reservoirs and ponds}

The water balance for large- and medium-sized reservoirs is represented explicitly by the reservoir module of SWAT2005 given that (1) they are of great importance to water supplies and (2) detailed characteristics are available for those reservoirs. The locations of the dams of those reservoirs are used to delineate sub-basins that are linked via the river network.

The pond module of SWAT2005 is improved in this paper. The large number of small-sized reservoirs and countless ponds (classes 1-3) are represented in the improved SWAT2005 in an aggregated manner (Fig. 2).

\section{Simplification and spatial distribution}

Because the geographic position of each small-sized reservoir is known, the spatial topology of small-sized reservoirs within an individual sub-basin is determined. According to a detailed survey of the spatial topology of small-sized reservoirs in individual sub-basins, it is reasonable to assume that small-sized reservoirs of the same class are interconnected in a parallel scheme, and small-sized reservoirs of different classes are interconnected in a sequential scheme (Fig. 2).

Given that the number, $n_{r}$, of water storages in each class $(r)$ is known for each sub-basin, the water balance of each water storage class $(r)$ within each sub-basin is calculated for a hypothetical representative reservoir, RM, with the mean characteristics for the water storage class within a sub-basin, i.e. with its storage capacity being equal to the mean value of the water storages belonging to that class located in the subbasin. The water balance of RM within each sub-basin was calculated with a daily time step using

$$
\begin{aligned}
V_{\mathrm{RM} r, \mathrm{t}} & =V_{\mathrm{RM} r, \mathrm{t}-1}+\frac{Q_{\mathrm{in}, r}}{n_{r}}-Q_{\mathrm{out}, \mathrm{RM} r}+V_{\mathrm{pcp}, \mathrm{RM} r} \\
& -V_{\text {evap,RM } r}-V_{\text {seep }, \mathrm{RM} r} r
\end{aligned}
$$

where $V_{\mathrm{RM} r \mathrm{t}}$ is the storage volume of the hypothetical reservoir (RM) in water storage class $r$ at day $t ; Q_{\mathrm{in}, r}$ is the daily inflow to water storage class $r ; n_{r}$ is the number of water storages in class $r ; Q_{\text {out,RM } r}$ is the daily outflow from reservoir $\mathrm{RM} ; V_{\mathrm{pcp}, \mathrm{RM} r}$ is the daily precipitation falling on the surface of reservoir RM; and $V_{\text {evap, RMr } r}$ and $V_{\text {seep, } \mathrm{RM} r}$ are the daily evaporation and seepage from reservoir RM, respectively (all in $\mathrm{m}^{3}$ ). The values of $V_{\mathrm{pcp}, \mathrm{RM} r}, V_{\text {evap, RM } r}$, and $V_{\text {seep, } \mathrm{RM} r}$ are calculated with SWAT2005, and the total actual storage volume $V_{r, \mathrm{t}}$ of water storage class $r$ within each sub-basin is obtained by

$V_{r, \mathrm{t}}=V_{\mathrm{RM} r, t} \cdot n_{r}$

\section{Inflow}

The total area of a sub-basin is divided into a number of runoff contributing areas, each corresponding to one water storage class. The inflow $Q_{\text {in, } r}$ of water storage class $r$ comprises direct inflow and additional inflow. The direct inflow to water storage class $r$ is the fraction of the total sub-basin runoff $Q_{\text {gen }}$ and is generated in a time step as the difference between the fraction $\mathrm{fr}_{r}$ of the sub-basin area that drains into water storage class $r$ and the fraction $\mathrm{fr}_{x}$ of the sub-basin area that drains into water storage class $x<r$ within the drainage of water storage class $r$, for example, the calculation of the inflow of class 2 described below. Additional inflow to a water storage class $r$ is provided by the fraction $\mathrm{fr}_{r}$ of outflow $Q_{\text {out }, x}$ of all water storage classes $x<r$ within the drainage of water storage class $r$. This approach accounts for the fact that a water storage could be located upstream of any larger water storage (not necessarily a water storage of the next larger class) and have no other smaller water storage in the downstream direction. Additionally, outflow from one water storage class is attributed to any larger water storage class within the sub-basin in the same time step.

\section{Inflow of class 1}

The pond category (class 1 ) is located on the top of the sequential and parallel routing scheme for water storages. The inflow of class 1 is the runoff generated in its drainage.

$Q_{\text {in, } 1}=\mathrm{fr}_{1} \cdot Q_{\text {gen }}$

\section{Inflow of class 2}

The inflow of class 2 is the sum of its direct inflow and additional inflow. The direct inflow is the difference between the 
runoff generated in the drainage of class 2 and that generated in the drainage of class 1 within the drainage of class 2 . The additional inflow is the outflow of class 1 within the drainage of class 2 .

$Q_{\text {in }, 2}=\left(1-\mathrm{fr}_{1}\right) \cdot \mathrm{fr}_{2} \cdot Q_{\text {gen }}+\mathrm{fr}_{2} \cdot Q_{\text {out }, 1}$

\section{Inflow of class 3}

Similar to the inflow of class 2, the direct inflow of class 3 is the difference between the runoff generated in the drainage of class 3 and that generated in the drainage of classes 1 and 2 within the drainage of class 3 . The additional inflow is the outflow of classes 1 and 2 within the drainage of class 3. However, the runoffs generated in the drainage of class 1 within the drainage of class 2 and the outflows of class 1 within the drainage of class 2 are not considered.

$$
\begin{aligned}
Q_{\text {in }, 3} & =\left(1-\mathrm{fr}_{1}-\mathrm{fr}_{2}+\mathrm{fr}_{1} \cdot \mathrm{fr}_{2}\right) \cdot \mathrm{fr}_{3} \cdot Q_{\mathrm{gen}} \\
& +\mathrm{fr}_{3} \cdot\left(Q_{\text {out }, 1}+Q_{\text {out }, 2}-\mathrm{fr}_{2} \cdot Q_{\text {out }, 1}\right)
\end{aligned}
$$

\section{Outflow}

Because classes 1-3 (small-sized reservoirs and ponds) are simulated with the pond module of SWAT2005, they are referred to as ponds below. In the pond module of SWAT2005, the volume of pond outflow may be calculated with the target storage approach. The target storage varies with flooding season and soil water content. The target volume is calculated as

$V_{\mathrm{tar}}=V_{\mathrm{em}}$ If $_{\text {mon }_{\text {fld,beg }}}<$ mon $<$ mon $_{\text {fld,end }}$

$$
\begin{aligned}
V_{\mathrm{tar}}= & V_{\mathrm{pr}}+\frac{\left(1-\min \left[\frac{\mathrm{sW}}{\mathrm{FC}}, 1\right]\right)}{2} \cdot\left(V_{\mathrm{em}}-V_{\mathrm{pr}}\right) \\
\quad \text { If } m o n & \leq \text { mon }_{\text {fld,beg }} \text { or mon } \geq \text { mon }_{\text {fld,end }}
\end{aligned}
$$

where $V_{\mathrm{tar}}$ is the target pond volume for a given day $\left(\mathrm{m}^{3}\right)$; $V_{\mathrm{em}}$ is the volume of water held in the pond when filled to the emergency spillway $\left(\mathrm{m}^{3}\right) ; V_{\mathrm{pr}}$ is the volume of water held in the pond when filled to the regular spillway $\left(\mathrm{m}^{3}\right)$; $\mathrm{SW}$ is the average soil water content in the sub-basin $(\mathrm{mm})$; FC is the water content of the sub-basin soil at field capacity (mm); mon is the month of the year; mon $_{\text {fld,beg }}$ is the beginning month of the flooding season; and mon fld,end $_{\text {is the ending }}$ month of the flooding season.

Once the target storage is defined, the pond outflow is calculated as

$V_{\text {flow out }}=\frac{V-V_{\text {tar }}}{\mathrm{ND}_{\text {tar }}}$

where $V_{\text {flow out }}$ is the volume of water flowing out of the pond during a day $\left(\mathrm{m}^{3}\right) ; V$ is the volume of water stored in the pond $\left(\mathrm{m}^{3}\right) ; V_{\mathrm{tar}}$ is the target pond volume for a given day $\left(\mathrm{m}^{3}\right)$; and $\mathrm{ND}_{\text {tar }}$ is the number of days required for the pond to reach its target storage.
The outflow regulations of ponds are not considered in the target storage calculation. Güntner et al. (2004) assumed that outflows from the small- and medium-sized reservoirs only occur if the storage capacities are exceeded. Because the small-sized reservoirs within the study area presented in Güntner et al. (2004) were mainly simple earth dams without any outflow regulation devices, the outflow calculations proposed by Güntner et al. (2004) were valid. However, in China, different outflow regulations are used in the different pond classes (classes 1-3) and during the different periods, including the non-flooding season, the beginning of the flooding season, the middle of the flooding season, and the end of the flooding season. Due to the lack of information on outflow regulations, in the target storage calculation, four regular storage volume adjustment parameters are set for the non-flooding season, the beginning of the flooding season, the middle of the flooding season, and the end of the flooding season.

\section{Target storage for class 1}

$V_{\mathrm{tar}}=V_{\mathrm{em}}$

\section{Target storage for classes 2 and 3}

$V_{\text {tar }}=\beta_{\text {fld,beg }} \cdot V_{\text {pr }}$ If May $<$ mon $<$ Jun

(the beginning of the flooding season)

$V_{\mathrm{tar}}=\beta_{\text {fld,mid }} \cdot V_{\mathrm{pr}}$ If Jul $<$ mon $<$ Aug

(the middle of the flooding season)

$V_{\mathrm{tar}}=\beta_{\mathrm{fld}, \mathrm{end}} \cdot V_{\mathrm{pr}}$ If Sept $<$ mon $<$ Oct

(the end of the flooding season)

$V_{\text {tar }}=\beta_{\text {nonflod }} \cdot V_{\mathrm{pr}}+\frac{\left(1-\min \left[\frac{\mathrm{sW}}{\mathrm{FC}}, 1\right]\right)}{2} \cdot\left(V_{\mathrm{em}}-V_{\mathrm{pr}}\right)$

If mon $\leq$ mon $_{\text {fld, beg }}$ or $\operatorname{mon} \geq$ mon $_{\text {fld,end }}$

where $V_{\mathrm{tar}}, V_{\mathrm{em}}, V_{\mathrm{pr}}, \mathrm{SW}, \mathrm{FC}$, mon, mon fld,beg , and mon $_{\text {fld,end }}$ have been described above, and $\beta_{\mathrm{fld}, \mathrm{beg}}, \beta_{\mathrm{fld} \text {,mid }}, \beta_{\mathrm{fld}, \text { end }}$, and $\beta_{\text {nonflod }}$ are the four regular storage volume adjustment parameters set for the beginning of the flooding season, the middle of the flooding season, the end of the flooding season, and the non-flooding season, respectively.

\subsubsection{Water-use data treatment based on the parameter calibration}

The water-use of each sector (including irrigation, livestock, domestic, industrial, and tourist water use) is distributed spatially according to land use and temporally based on weather conditions (e.g. annual precipitation). Referring to the sources of water uses (including water storages, river reaches, and shallow aquifers), six fraction parameters are defined for two separate time periods (October to April and 
May to September), including, $\alpha_{\text {pnd,octapr }}$ and $\alpha_{\text {pnd,maysep }}$ for water storages, $\alpha_{\text {rch,octapr }}$ and $\alpha_{\text {rch,maysep }}$ for river reaches, and $\alpha_{\mathrm{gw}, o c t a p r}$ and $\alpha_{\mathrm{gw} \text {,maysep }}$ for shallow aquifers, in the period of October to April and the period of May to September, respectively.

\subsection{Calibrating physical parameters and human interference parameters}

\subsubsection{Calibration approach}

The physical parameter calibration is first processed for the case with minimal human activities. The human interference parameter calibration is then processed with significant human activities. Because distributed, extensive water storage within a basin can significantly influence the basin hydrologic processes, and the impacts must then be included in the parameterization and calibration of a hydrological simulation model, by distinguishing these two stages, we apply the parameters calibrated during the first stage to the hydrological simulation in the second stage, so as to avoid the equifinality problem of parameters (Cibin et al., 2009). In this paper, the sensitivity analysis tool and the calibration helper module of SWAT2005 are used to calibrate the parameters during the two stages.

\subsubsection{Evaluation criterion}

The mean relative error (MRE), the coefficient of determination $\left(R^{2}\right)$, and the Nash-Sutcliffe efficiency (NSE) are used to evaluate the simulated streamflows with the observed streamflows.

The MRE is computed according to Eq. (21).

$\mathrm{MRE}=\frac{P^{\text {mean }}-Q^{\text {mean }}}{Q^{\text {mean }}} \times 100 \%$

where $P^{\text {mean }}$ and $Q^{\text {mean }}$ are the means of the simulated streamflows and the observed streamflows, respectively. "MRE values of 0 indicate a perfect fit. Positive values indicate model overestimation bias, and negative values indicate model underestimation bias (Hao et al., 2006)."

$R^{2}$ is computed according to Eq. (22).

$R^{2}=\frac{\sum_{i=1}^{n}\left(P_{i}-P^{\text {mean }}\right)\left(Q_{i}-Q^{\text {mean }}\right)}{\sqrt{\sum_{i=1}^{n}\left(P_{i}-P^{\text {mean }}\right)^{2} \sum_{i=1}^{n}\left(Q_{i}-Q^{\text {mean }}\right)^{2}}}$

where $P_{i}$ is the $i$ th simulated value for the streamflows; $Q_{i}$ is the $i$ th observation for the streamflows; $P^{\text {mean }}$ is the mean of the simulated streamflows; $Q^{\text {mean }}$ is the mean of the observed streamflows; and $n$ is the total number of observations. " $R^{2}$ describes the portion of the variance in measured data explained by the model. $R^{2}$ ranges from 0 to 1 , with lower values indicating more error variance, and typically $R^{2}=1$ is considered the optimal value (Moriasi et al., 2007)."
NSE is computed according to Eq. (23).

$$
\mathrm{NSE}=1-\frac{\sum_{i=1}^{n}\left(Q_{i}-P_{i}\right)^{2}}{\sum_{i=1}^{n}\left(Q_{i}-Q^{\text {mean }}\right)^{2}}
$$

where $Q_{i}$ is the $i$ th observation for the streamflows; $P_{i}$ is the $i$ th simulated value for the streamflows; $Q^{\text {mean }}$ is the mean of the observed streamflows; and $n$ is the total number of observations. The NSE ranges between $-\infty$ and 1.0, with NSE $=1.0$ as the optimal value. According to Luo et al. (2008), values between 0.0 and 1.0 are generally viewed as acceptable levels of performance, whereas a value less than 0.0 means that the mean observed value is a better predictor than the simulated value, which indicates unacceptable performance.

\section{Datasets}

\subsection{Study site}

This study is applied to the basin with the Fengman Reservoir, which has a storage volume of more than $112 \times 10^{8} \mathrm{~m}^{3}$, is located in the Second Songhua River, situated in the southeast of Jilin province in China. The basin drains an area of $42500 \mathrm{~km}^{2}$, occupying $55 \%$ of the total drainage area of the Second Songhua River and consisting of approximately 2000 reservoirs and countless ponds. Approximately 9335 water storages, each with a water area of more than $4000 \mathrm{~m}^{2}$, could be identified in the basin with the 2002 satellite remote sensing images. The impoundment and release of these storages have a significant influence on the inflows to the Fengman Reservoir, thus making the Fengman Reservoir increasingly difficult to be operated, particularly during flooding seasons. Meanwhile, during non-flooding seasons and the preliminary stage of a flooding season, inflows to the Fengman Reservoir are reduced due to the impoundment of water storages, and power generation is affected. In the middle of flooding seasons, inflows to the Fengman Reservoir increase due to the release of water storages, and flood control is affected. In 1995, due to the impoundment and release of water storages, the unpredictable inflows to the Fengman Reservoir were approximate $4 \times 10^{8} \mathrm{~m}^{3}$ during the flooding season. There are so many small- and medium-sized water storages in the upper Fengman Reservoir Basin that it is difficult to obtain their detailed design and running information. Furthermore, computing time increases substantially when all of the water storages in the upper Fengman Reservoir Basin are added to the models. Therefore, the basin hydrologic water cycle simulation is becoming increasingly difficult, and the impact rules of the impoundment and release of water storages on runoff are difficult to obtain.

The study area is limited to the upper-middle stream region of the Fengman Reservoir Basin above the Wudaogou 


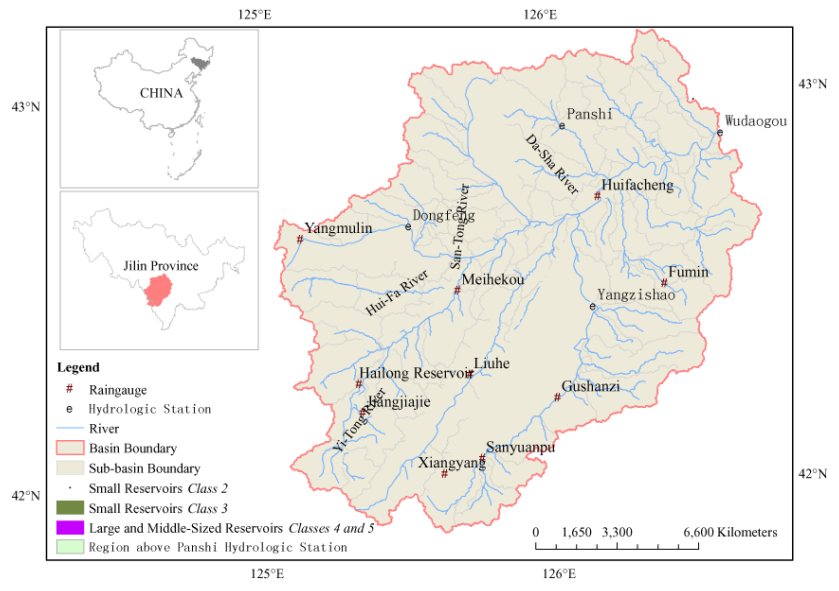

Fig. 3. The Fengman Reservoir Basin.

hydrologic station, although it is still referred to as the Fengman Reservoir Basin in the rest of this study. The basin drains an area of $12411 \mathrm{~km}^{2}$. Its mean annual precipitation is $720 \mathrm{~mm}$, and its mean annual precipitation during the flooding season is $510 \mathrm{~mm}$, accounting for more than $70 \%$ of its mean annual precipitation. The water storages within the basin drain an area of $7421.27 \mathrm{~km}^{2}$, accounting for $63.98 \%$ of the basin drainage area.

Ten rain gauges (Liuhe, Huifacheng, Fumin, Hailong, Sanyuanpu, Xiangyang, Meihekou, Gushanzi, Jiangjiajie, and Yangmulin) and four hydrologic stations (Panshi, Dongfeng, Yangzishao, and Wudaogou) are within the basin (Fig. 3). The ten rain gauges provide daily precipitation data, whereas the four hydrologic stations provide daily precipitation and streamflow data. The spatial topology of water storages in the individual sub-basin above the Panshi hydrologic station (Fig. 4) justifies the assumptions that smallsized reservoirs of the same class are interconnected in a parallel scheme and that small-sized reservoirs of different classes are interconnected in a sequential scheme.

Based on the survey, one large-sized reservoir (class 5), i.e. the Hailong reservoir, twelve middle-sized reservoirs (class 4), approximately 500 small-sized reservoirs (classes 2 and 3), and countless ponds (class 1) were built from the 1950s to the 1980s in the Fengman Reservoir Basin. The total storage volume of classes $2-5$ is approximately $9.58 \times 10^{8} \mathrm{~m}^{3}$. The storage volume of the Hailong Reservoir is approximately $3.70 \times 10^{8} \mathrm{~m}^{3}$. The total storage volume of classes $2-4$ is approximately $5.88 \times 10^{8} \mathrm{~m}^{3}$. Therefore, the numerous water storages significantly influence the runoff within the basin. The drainage area and storage volume of each water storage class within the basin are shown in Table 1. Numerous water storages were built from the 1960s to the 1990s, and a few water storages were built before 1956 or after 1990. Therefore, the pre-1956 and post-1990 periods are treated as the first (with natural condition only) and the second stage (with human interference), respectively.

\subsection{Data collection}

The data sources and processing methods are described as follows for the application of the improved SWAT2005.

i. DEM data (raster resolution: $90 \mathrm{~m} \times 90 \mathrm{~m}$ ), obtained from the International Scientific Data Service Platform of the Computer Network Information Center, Chinese Academy of Sciences (http://srtm.csi.cgiar.org).

ii Soil data $\left(\right.$ scale $\left.=1: 10^{6}\right)$, collected from the Data Center for Resources and Environmental Sciences Chinese Academy of Sciences (RESDC).

iii. Land use data for the 1980 s and 2000s (scale $=1: 10^{5}$ ), collected from the Data Center for Resources and Environmental Sciences Chinese Academy of Sciences (RESDC). Because several water storage facilities were built before 1956 or after 1990 in the Fengman Reservoir Basin, basin land use data for the years before 1956 and after 1990 are also needed. Previous studies show that from 1954 to 1976, the main land use change was converting some forest area to crop and grass lands in the upstream mountain area (Zhang et al., 2006; Kuang et al., 2006). The population of the eastern mountain area of Jilin Province increased from 1950s to 2000s by about $160 \%$. To obtain basin land use data for the years before 1956, the basin land use data for the 1980s, which is available to this study, are modified as follows: (1) the basin water storage land use is modified to forest, and (2) according to the basin population distribution, the basin grass and crop land uses in the locations, where the population in 1980 is dense but in 1956 is sparse, are modified to forest.

iv. Digital river network data $\left(\right.$ scale $\left.=1: 2.5 \times 10^{5}\right)$, obtained from the 1:4 M-scale Topographic Database of the National Fundamental Geographic Information System of China.

v. Daily precipitation data for 14 stations over a 54yr period (1953-2006) and daily streamflow data for 4 stations over a 53-yr period (1954-2006), obtained from the Hydrological Administration of Jilin Province, China; daily meteorological data (temperature, solar radiation, weed speed, and relative humidity) for 4 stations over a 54-yr period (1953-2006), obtained from the China Meteorological Data Sharing Service System.

vi. Individual water storage characteristics, obtained from the Hydrological Administration of Jilin Province, China. Detailed design and running information are known for the medium- and large-sized reservoirs (classes 4 and 5). For small-sized reservoirs (classes 2 and 3), only their geographic positions, drainage areas, emergency storage volumes, and regular storage volumes are available at the level of administrative 
Table 2. Classification of water storages and correlations between surface area $\left(A ; \mathrm{m}^{2}\right)$ and regular storage volume $\left(V ; 10^{4} \mathrm{~m}^{3}\right)$ of each water storage class within the basin.

\begin{tabular}{clll}
\hline \multicolumn{3}{c}{ Classification indices } & \\
\cline { 1 - 3 } Slope (\%) & $\begin{array}{l}\text { Drainage area } \\
\left(\mathrm{km}^{2}\right) / \text { regular } \\
\text { storage volume } \\
\left(10^{4} \mathrm{~m}^{3}\right)\end{array}$ & $\begin{array}{l}\text { Regular } \\
\text { storage } \\
\text { volume } \\
\left(10^{4} \mathrm{~m}^{3}\right)\end{array}$ & $\begin{array}{l}\text { Correlation } \\
\text { coefficient } \\
\text { between } \\
A \text { and } V\end{array}$ \\
\hline \multirow{5}{*}{$0 \sim 15$} & $>=0.14$ & $>=10$ & \\
& $<0.14$ & $0 \sim 10$ & 0.80 \\
& & $>=10$ & 0.74 \\
\hline & $>=0.1$ & $<=17$ & 0.90 \\
& & $17 \sim 77$ & 0.63 \\
& $<0.1$ & $>=77$ & 0.73 \\
& & $<77$ & 0.63 \\
& & $>=77$ & 0.73 \\
\hline
\end{tabular}

units (municipalities). For ponds (class 1), only the total drainage area and storage volume are known at the basin level.

vii. Water use data for the 2000s, obtained from the Hydrological Administration of Jilin Province, China, and used as a baseline.

\section{Results and discussions}

The classifications and relationships between surface area and regular storage volume for each of the water storage classes within the basin are obtained using the aforementioned methods, as shown in Table 2. It is obvious that water storages with different slopes, storages, and drainage areas have different relationships between surface area and storage volume. Because the values of $R^{2}$ exceed 0.6 , the obtained relationships between surface area and storage volume of water storages are statistically reliable. The improved SWAT2005 is used to simulate streamflow in the Fengman Reservoir Basin. Because hydrologic stations within the basin are scarce in the streamflow data for the 1950s, the Yangzishao and Wudaogou hydrologic stations were chosen as calibration stations during the first stage with natural conditions, and the streamflow data from the pre-1960 period were used to calibrate the physical parameters. Because water storages are the main human activities taking place in the upper part of the Panshi and Dongfeng hydrologic stations, the Panshi and Dongfeng hydrologic stations were chosen as calibration stations during the second stage, and the streamflow data from the 1990-1995 and post-1996 periods were used to calibrate and validate the human interference parameters, respectively. The Yangzishao and Wudaogou hydrologic stations were chosen as the improved SWAT2005 validation stations during the second stage, and the streamflow

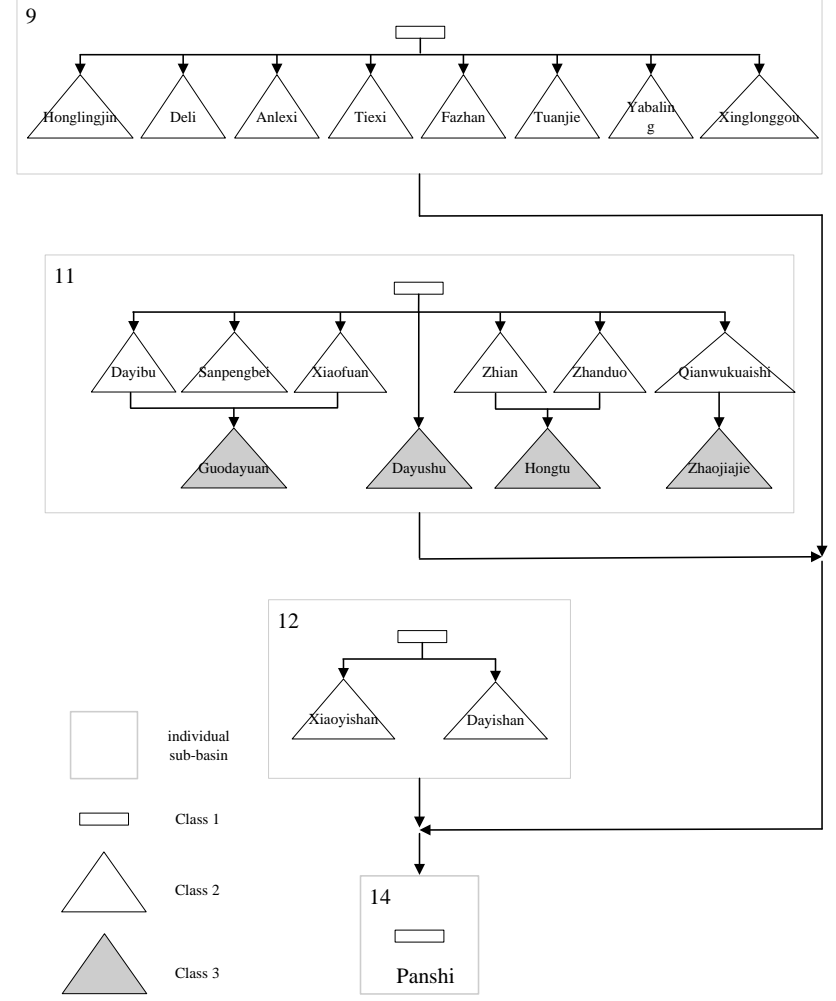

Fig. 4. Spatial topology of the water storages in the Panshi sub-basin Additionally, "9", "11", "12" and "14" in rectangles (individual subbasins) mean the numbers of individual sub-basins.

data from the post-1990 period were used to validate the improved SWAT2005.

To compare the performance between the original and improved SWAT2005, two scenarios, S0 (simulating human activities using the original SWAT2005 with the calibrated physical parameters) and S1 (simulating human activities using the improved SWAT2005 with the aforementioned calibrated physical and human interference parameters described), are designed. To examine the performance of water balance and transport through a network combining both sequential and parallel streams and storage links in the improved SWAT2005, an additional scenario, S2 (modelling water balance and transport through a network combining both sequential and parallel streams and storage links, while ignoring the human interference parameters), is designed.

\subsection{Calibration results}

Figure 5 shows the observed and simulated monthly streamflows during the physical parameter calibration period (before 1960) at the Yangzishao and Wudaogou hydrologic stations, and the calibrated physical parameters are shown in Table 3. The values of $R^{2}$ and NSE exceed 0.6 and 0.8 , respectively, and the value of MRE does not exceed $10 \%$ in the first-stage calibration. Therefore, the first-stage calibration 


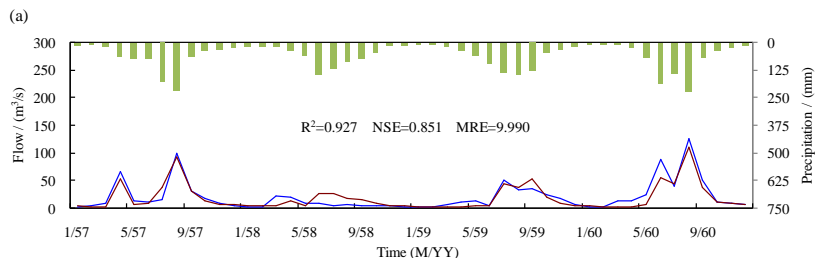

(b)

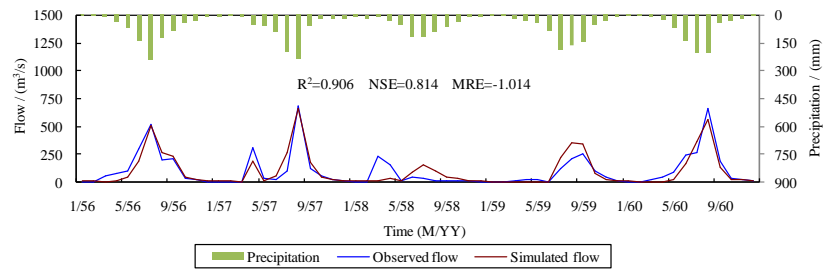

Fig. 5. Observed and simulated monthly streamflows during the physical parameter calibration period (before 1960) at two discharge gauges: (a) Yangzishao and (b) Wudaogou, where $R^{2}$ is the coefficient of determination; NSE is the Nash-Sutcliffe efficiency; and MRE (\%) refers to the mean relative error.

Table 3. Calibrated physical parameters.

\begin{tabular}{|c|c|c|}
\hline Parameter Nam & Original Value & Calibrated Value \\
\hline ALPHA_BF & 0.048 & 0.477 \\
\hline ESCO & 0.950 & 0.968 \\
\hline GW_DELAY & 31.000 & 5.541 \\
\hline SFTMP & 1.000 & 0.73 \\
\hline SMTMP & 0.500 & 4.441 \\
\hline SMFMX & 4.500 & 3.136 \\
\hline TIMP & 1.000 & 0.048 \\
\hline GWQMN & 0.000 & 20.000 \\
\hline RCHRG_DP & 0.050 & 0.100 \\
\hline & & 0.756 \\
\hline \multirow{3}{*}{\multicolumn{2}{|c|}{ CANMX }} & 2.8 (crop) \\
\hline & & 4.8 (forest) \\
\hline & & 4.1 (grass) \\
\hline
\end{tabular}

a Parameters are multiplicative factors used to simultaneously adjust all spatially variable base values of the $\mathrm{CN} 2$ parameter.

results meet the precision requirements for simulating the basin hydrologic cycle.

Figure 6 shows the observed and simulated monthly streamflows by $\mathrm{S} 0$ and $\mathrm{S} 1$ during the second-stage calibration considering human interferences (1990-1995) at the Panshi and Dongfeng hydrologic stations. The calibrated human interference parameters are shown in Table 4 . The values of $R^{2}$ and NSE exceed 0.6 and 0.8 , respectively, and the value of MRE does not exceed $10 \%$ in the second-stage calibration. Therefore, the second-stage calibration results also meet the precision requirements for simulating the basin hydrologic cycle.
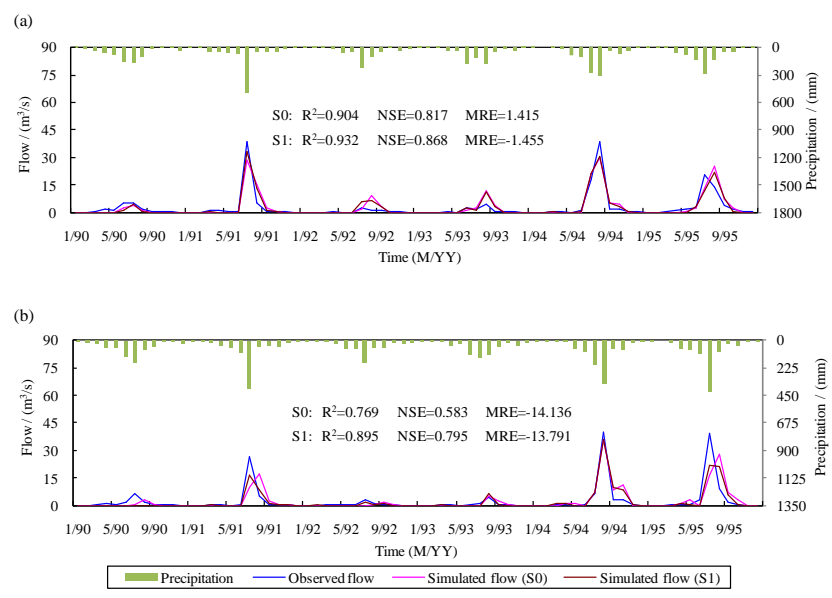

Fig. 6. Observed and simulated monthly streamflows during the human interference parameter calibration period (1990-1995) at two discharge gauges: (a) Panshi and (b) Dongfeng. S0 refers to the consideration of human activities by the original SWAT2005 with calibrated physical parameters, while $\mathrm{S} 1$ refers to the consideration of human activities by the improved SWAT2005 with the calibrated physical and human interference parameters described above.

\subsection{Validation results}

Figure 7 shows the observed and simulated monthly streamflows by $\mathrm{S} 0$ and $\mathrm{S} 1$ over the validation periods at the Panshi, Dongfeng, Yangzishao and Wudaogou hydrologic stations. There is a significant improvement in the simulation at the Panshi and Dongfeng hydrologic stations, while the improvement of the simulation at the Yangzishao and Wudaogou hydrologic stations is limited. Over the validation periods, the mean model performance rises from $0.887\left(R^{2}\right)$ and 0.735 (NSE) to 0.925 and 0.848 at the Panshi and Dongfeng hydrologic stations, respectively, while the mean model performance rises from $0.922\left(R^{2}\right)$ and 0.843 (NSE) to 0.964 and 0.913 at the Yangzishao and Wudaogou hydrologic stations, respectively.

As shown in Fig. 7, there are six flooding events in the validation periods at Panshi and Dongfeng Stations, which occurred in 1996, 1998, 2003, 2004, 2005, and 2006, respectively; while there are eleven flooding events in the validation periods at Yangzishao and Wudaogou Stations, occurring in 1991, 1993, 1994, 1995, 1996, 1998, 2001, 2003, 2004, 2005, and 2006, respectively. Because both large floods and small floods are needed to demonstrate the performance of the model in the flooding season, we choose the 1998 and 2004 flooding seasons at the Panshi and Dongfeng hydrologic stations, the 1995 and 2004 flooding seasons at the Yangzishao and Wudaogou hydrologic stations. Figure 8 shows the evaluation criterion for the flooding seasons over the validation periods, the observed and simulated monthly streamflows by S0 and S1 for the 1998 and 2004 flooding seasons at the Panshi and Dongfeng hydrologic stations, as 
Table 4. Calibrated human interference parameter values, including the regular storage volume adjustment parameters and the fraction parameters for different water sources.

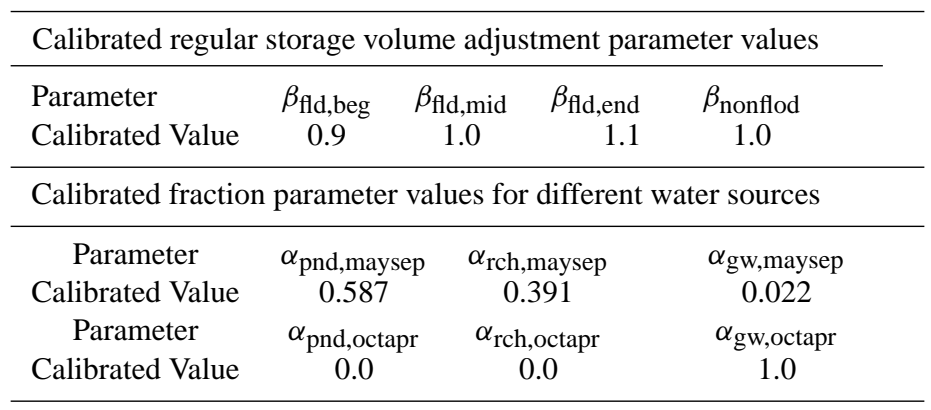
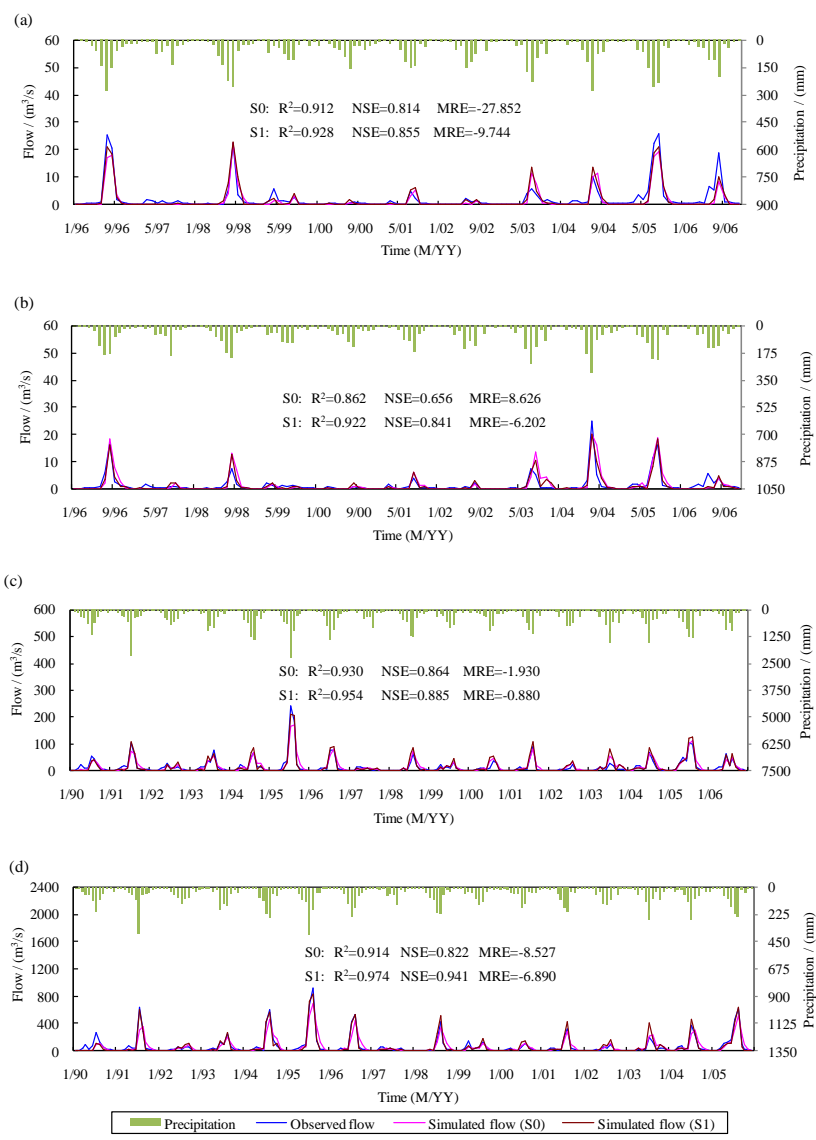

Fig. 7. Observed and simulated monthly streamflows over the validation periods at all four discharge gauges: (a) Panshi (19962006), (b) Dongfeng (1996-2006), (c) Yangzishao (1990-2006) and (d) Wudaogou (1990-2006).

well as the observed and simulated monthly streamflows by S0 and S1 for the 1995 and 2004 flooding seasons at the Yangzishao and Wudaogou hydrologic stations. Figure 8 also shows a clear improvement in the simulation at the Panshi and Dongfeng hydrologic stations, while the simulation at the Yangzishao and Wudaogou hydrologic stations is improved less significantly for the flooding seasons over the validation periods. For the flooding seasons over the validation periods, the mean model performance rises from 0.878 $\left(R^{2}\right)$ and 0.719 (NSE) to 0.921 and 0.843 at the Panshi and Dongfeng hydrologic stations, respectively, while the mean model performance rises from $0.929\left(R^{2}\right)$ and 0.826 (NSE) to 0.961 and 0.910 at the Yangzishao and Wudaogou hydrologic stations, respectively.

Figure 9 compares the evaluation criteria of S0, S1, and $\mathrm{S} 2$ for the flooding seasons over the validation periods at the Panshi and Dongfeng hydrologic stations. For the flooding seasons over the validation periods, the mean model performance rises from $0.878\left(R^{2}\right)$ and 0.719 (NSE) by S0 to 0.907 and 0.818 by $\mathrm{S} 2$ at the Panshi and Dongfeng hydrologic stations, respectively, and the $R^{2}$ and NSE in S2 are improved by $66.824 \%$ and $80.114 \%$, respectively, compared to those in $\mathrm{S} 1$.

Figure 10 shows the water balance and transport through a network combining both sequential and parallel streams and storage links above the Panshi hydrologic station over the validation periods. The results indicate that approximately $9.0 \%$ of the annual total inflows to small-sized reservoirs (class 2) are derived from ponds (class 1), and approximately $9.2 \%$ and $7.7 \%$ of the annual total inflows to small-sized reservoirs (class 3) are derived from ponds (class 1) and small-sized reservoirs (class 2), respectively. The annual water supplies from ponds (class 1), smallsized reservoirs (class 2) and small-sized reservoirs (class 3) are $9.79 \times 10^{6} \mathrm{~m}^{3}, 6.62 \times 10^{6} \mathrm{~m}^{3}$, and $9.39 \times 10^{6} \mathrm{~m}^{3}$, respectively.

\subsection{Discussions}

The results indicate that the simulation accuracy is improved at all four hydrologic stations in the improved SWAT2005 compared to the original SWAT2005. However, the improvements are more significant in the flooding season and vary by location in the watershed. The improvement is significant in the simulation of the Panshi and Dongfeng hydrologic stations, while it is limited for the Yangzishao and $\mathrm{Wu}$ daogou hydrologic stations over the validation periods. The same observation is found with the flooding seasons over the 

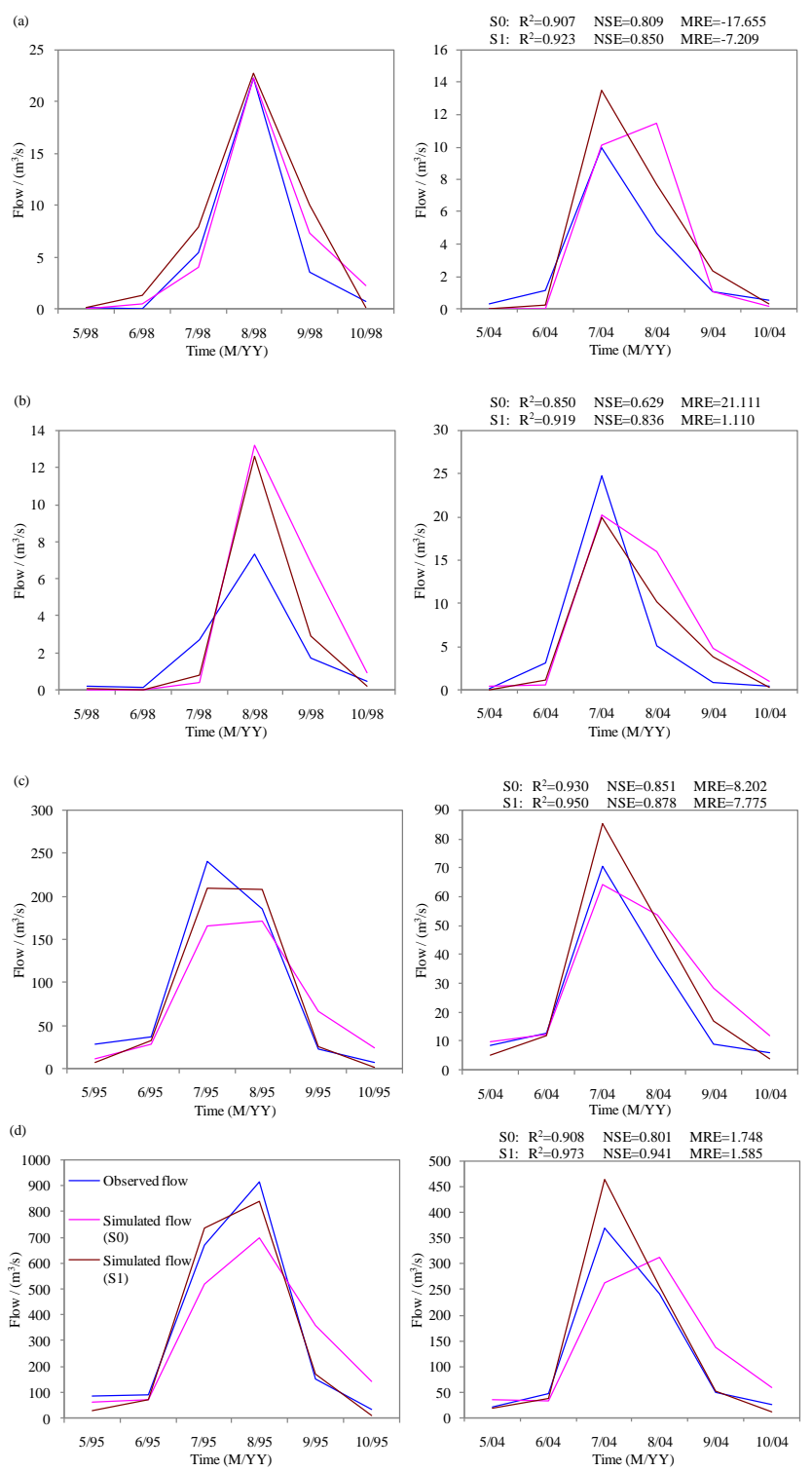

Fig. 8. Observed and simulated monthly streamflows for the 1998 and 2004 flooding seasons at two discharge gauges: (a) Panshi and (b) Dongfeng, and observed and simulated monthly streamflows for the 1995 and 2004 flooding seasons at two discharge gauges: (c) Yangzishao and (d) Wudaogou, where the evaluation criteria $\left(R^{2}\right.$, NSE, and MRE) within each sub-figure refer to all of the flooding seasons over the validation periods.

validation periods. The Panshi and Dongfeng hydrologic stations are located in the upper stream region of the Fengman Reservoir Basin, and the water storages reflect the major human activities within their drainages. While the Yangzishao and Wudaogou hydrologic stations are located in the lower stream region of the Fengman Reservoir Basin, and the water storages only represent part of human activities, it is difficult to take the complicated human activities into account when simulating the hydrologic cycle within their drainages.
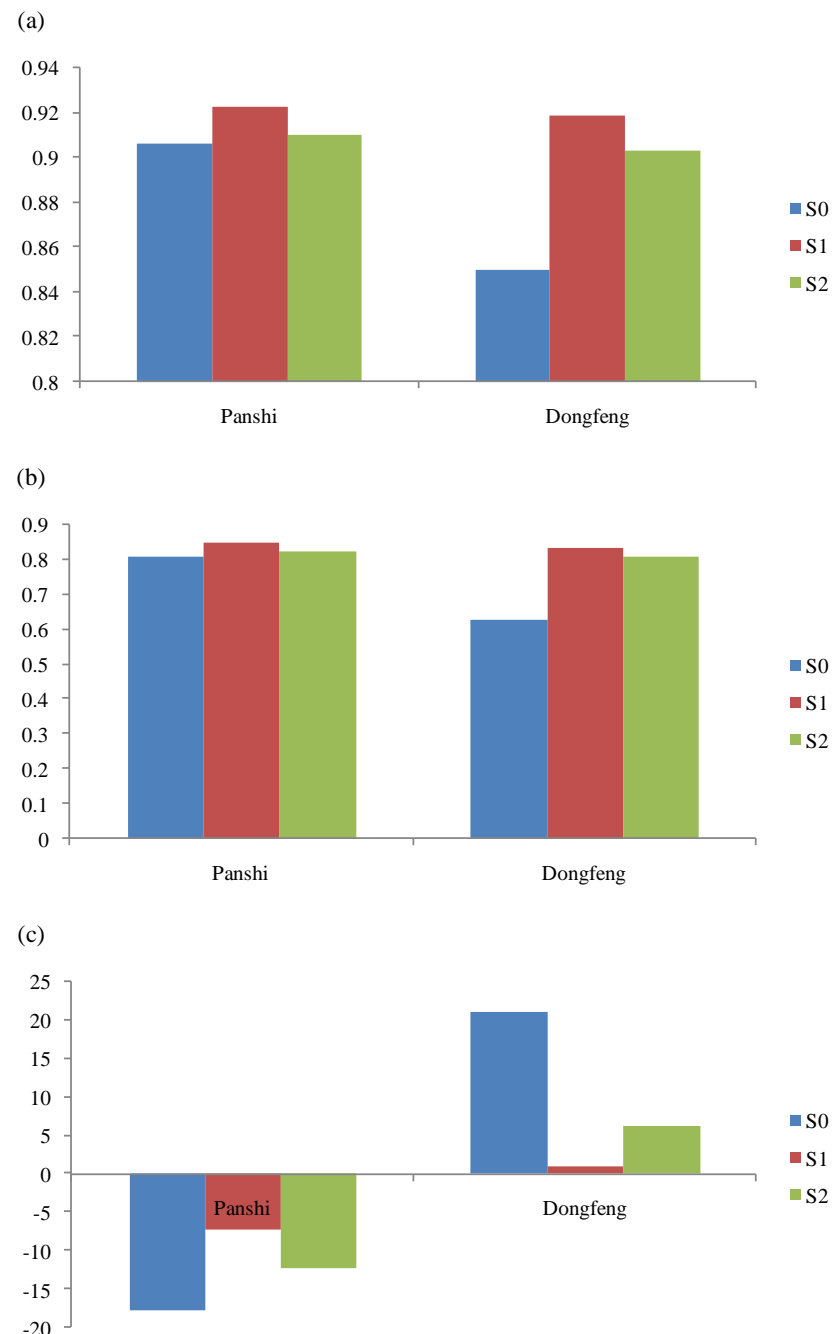

Fig. 9. Comparisons of the evaluation criterions for the flooding seasons over the validation periods: (a) $R^{2}$, (b) NSE and (c) MRE. In $\mathrm{S} 2$, considering the water balance and transport through a network combining both sequential and parallel streams and storage links while ignoring the human interference parameters.

Therefore, the simulation accuracy is improved more at the Panshi and Dongfeng hydrologic stations. The improvements over the validation periods are mostly due to the improvements in the flooding seasons over the validation periods, and the improvements in the flooding seasons over the validation periods are mainly due to the consideration of the water balance and transport through a network combining both sequential and parallel streams and storage links. 

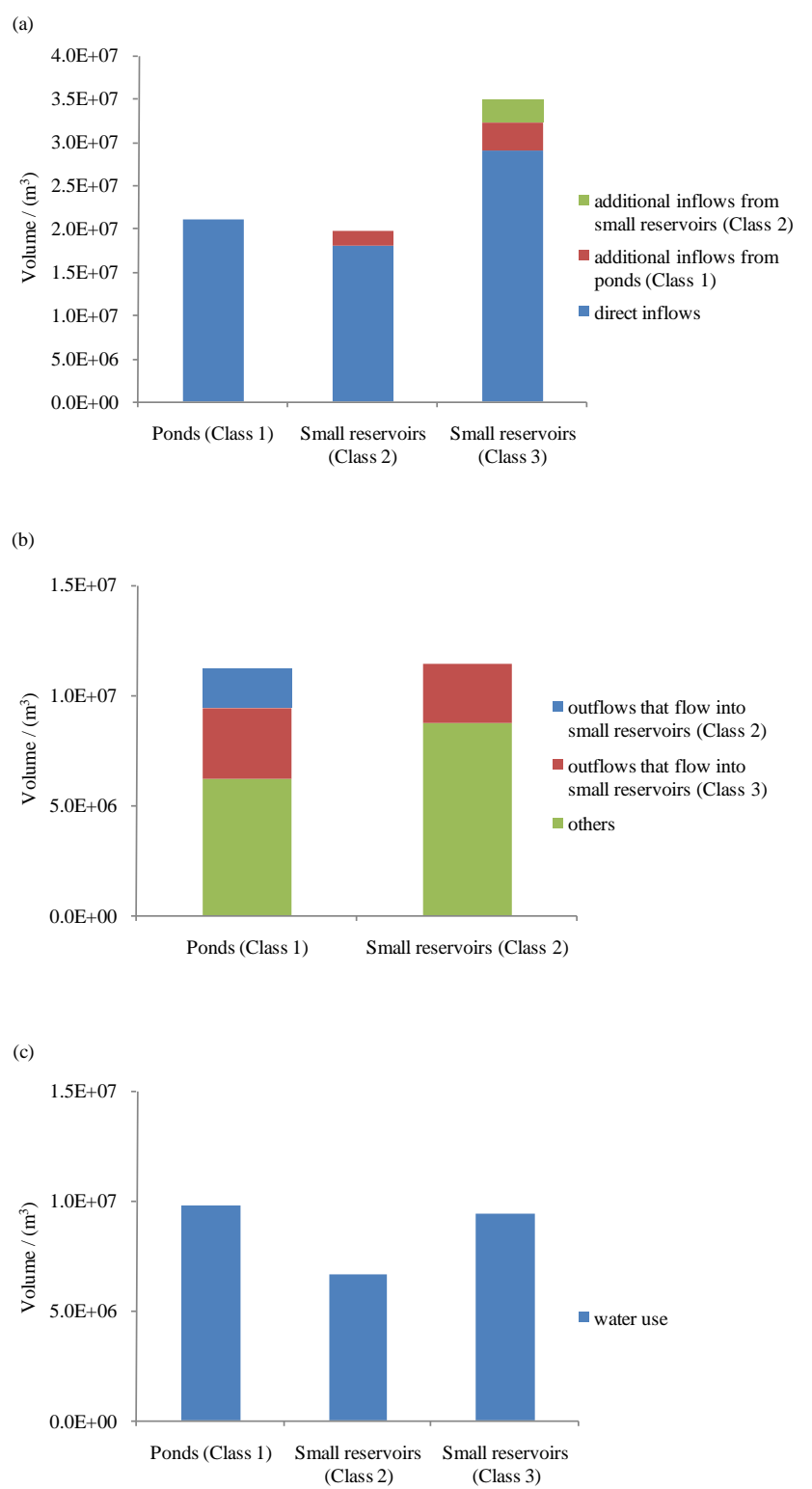

Fig. 10. Water balance and transport through a network combining both sequential and parallel streams and storage links above Panshi hydrologic station over the validation periods: (a) Inflow, (b) Outflow and (c) Water Use.

\section{Conclusions}

SWAT2005 has been improved in the following aspects: (1) a realistic representation of the relationships between the surface area and volume of each type of water storages, ranging from small-sized ponds for water flow regulation to medium- and large-sized reservoirs for water supply, hydropower generation, etc.; (2) water balance and transport through a network combining both sequential and parallel streams and storage links; and (3) calibrations for both physical and human interference parameters (conducted in two stages, i.e. without and with human activities). Through a real-world watershed case study, it is found that the improved SWAT2005 more accurately models small- and mediumsized storages than the original model in reproducing streamflows, especially in flooding seasons and in the basins where water storages represent major human activities.

A two-stage parameter calibration strategy and three scenarios are used to examine the performance between the original and improved SWAT2005 in the regions with numerous small- and medium-sized water storages. Compared to the original SWAT2005, the simulation accuracy during the stage with human interference is improved at each hydrologic station with the improved SWAT2005. The results indicate that water balance and transport through a network combining both sequential and parallel streams and storage links is modeled with a reasonable accuracy, especially in the flooding seasons, by the improved SWAT2005. By calibrating both the physical and human interference parameters, the observed and simulated flows match better than calibrating with the physical parameters only. The improved SWAT2005 can be used in other basins, particularly those basins with extensive water storages.

Several limitations exist with this study and can be solved by future work. The validation of the improved SWAT2005 and the calibration of its simulated streamflows are only processed with limited discharge gauges. The simulation results within the drainages of the limited discharge gauges could be better calibrated and validated with more gauge stations. The design of the water balance and transport through a network combining both sequential and parallel streams and storage links is subject to further validation, although it works well with the case study basin present in this study since it is able to represent the basic mechanisms of hydrological processes and water management that influence the water balance, while being flexible enough to allow for inclusions of additional process knowledge or data. With better data on topography, locations and surface area variations of reservoirs from remote sensing studies, as well as the reservoir operation rules, the improved SWAT2005 and its validation approach could be refined.

Acknowledgements. We are pleased to acknowledge the financial support for this project from the National Natural Science Foundation of China entitled Research on Laws of the Impacts of Human Activities on Catchment Water Cycle Based on SWAT Model (Grant No. 51079014) and the National Natural Science Foundation of China entitled Hydropower Reservoir Optimal Operation under Hydrometeorological Uncertain Enviroment (Grant No. 51109025). We would also like to thank the Institute of Water Resources and Flood Control of Dalian University of Technology in China for providing the data essential for this research.

Edited by: N. Basu 


\section{References}

Arnold, J. G. and Fohrer, N.: SWAT2000: current capabilities and research opportunities in applied watershed modelling, Hydrolog. Process., 19, 563-572, 2005.

Borah, D. K. and Bera, M.: Watershed-scale hydrologic and nonpoint-source pollution models: review of applications, Trans. ASAE, 47, 789-803, 2004.

Cao, B.: Research on reservoir dynamic capacity based on remote sensing and DEM, Dissertation of Doctoral Degree. Wuhan: Huazhong University of Science and Technology, 2006.

Chorography Editorial Committee of Jilin Province, Jilin Province Chorography Population, Changchun: Jilin People's Publishing House, 1991.

Cibin, R., Sudheer, K. P., and Chaubey, I.: Sensitivity and identifiability of stream flow generation parameters of the SWAT model, Hydrolog. Process., 24, 1133-1148, 2009.

Ding, Z. X.: Study on method for measurement of reservoir waterlevel and water surface curve based on DEM combined with remote sensing, Water Resour. Hydrop. Eng., 41, 83-86, 2012.

Du, Y. Y. and Zhou, C. H.: Automatically extracting remote sensing information for water bodies, J. Remote Sens., 2, 264-269, 1988.

Frazier, P. S. and Page, K. J.: Water body detection and delineation with Landsat TM data, ISPRS J. Photogramm. Eng. Remote Sens., 66, 1461-1467, 2006.

Gao, B. C.: NDWI-A normalized difference water index for remote sensing of vegetation liquid water from space, Remote Sens. Environ., 58, 257-266, 1995.

Gardini, B., Graf, G., and Ratie, G.: The instruments on Envisat, Acta Astronaut., 37, 301-311, 1995.

Gassman, P. W., Reyes, M. R., Green, C. H., and Arnold, J. G.: The soil and water assessment tool: historical development, applications, and future research directions, Trans. ASABE, 50, 12111250, 2007.

Gross, E. J. and Moglen, G. E.: Estimating the hydrological influence of Maryland state dams using GIS and the HEC-1 model, J. Hydrol. Eng., 12, 690-693, 2007.

Güntner, A., Krol, M. S., De Araújo, J. C., and Bronstert, A.: Simple water balance modelling of surface reservoir systems in a large data-scarce semiarid region, Hydrol. Sci. J., 49, 901-918, 2004.

Hao, F., Cheng, H., and Yang, S.: Non-point source pollution model, China Environmental Science Press, Beijing, 2006.

Jayatilaka, C. J., Sakthivadivel, R., Shinogi, Y., Makin, I. W., and Witharana, P.: A simple water balance modelling approach for determining water availability in an irrigation tank cascade system, J. Hydrol., 273, 81-102, 2003.

Kuang, W. H., Zhang, S. W., Zhang, Y. Z., Li, Y., and Hou, W.: Changes of forest landscape and its driving mechanism during the last fifty years in the eastern mountain area of Jilin Province, Journal of Beijing Forestry University, 28, 38-45, 2006.

Li, Y. S. and Wu, P. F.: Study on the changes in Ebinur lake based on the MODIS data, J. Water Res. Water Eng., 19, 110-112, 2008.

Liebe, J. R., Van De Giesen, N., and Andreini, M.: Estimation of small reservoir storage capacities in a semi-arid environment. A case study in the upper east region of Ghana, Phys. Chem. Earth, 30, 448-454, 2005.
Liebe, J. R., Van De Giesen, N., Andreini, M., Walter, M. T., and Steenhuis, T. S.: Determining watershed response in data poor environments with remotely sensed small reservoirs as runoff gauges, Water Resour. Res., 45, W07410, doi:10.1029/2008WR007369, 2009.

Lopez-Moreno, J. I., Vicente-Serrano, S. M., Begueria, S., GarciaRuiz, J. M., and Portela, M. M.: Dam effects on droughts magnitude and duration in a transboundary basin: the Lower River Tagus, Spain and Portugal, Water Resour. Res., 45, W02405, doi:10.1029/2008WR007198, 2009.

Luo, Y., He, C., Sophocleous, M., Yin, Z., Ren, H., and Zhu, O.: Assessment of crop growth and soil water modules in SWAT2000 using extensive field experiment data in an irrigation district of the Yellow River Basin, J. Hydrol., 352, 139-156, 2008.

McFeeters, S. K.: The use of the normalized difference water index (NDWI) in the delineation of open water features, Int. J. Remote Sens., 17, 1425-1432, 1996.

Mialhe, F., Gunnell, Y., and Mering, C.: Synoptic assessment of water resource variability in reservoirs by remote sensing: General approach and application to the runoff harvesting systems of south India, Water Resour. Res., 44, W05411, doi:10.1029/2007WR006065, 2008.

Moriasi, D. N., Arnold, J. G., Van Liew, M. W., Bingner, R. L., Harmel, R. D., and Veith, T. L.: Model evaluation guidelines for systematic quantification of accuracy in watershed simulations, Transactions of the American Society of Agricultural and Biological Engineers, 50, 885-900, 2007.

Neitsch, S. L., Arnold, J. G., Kiniry, J. R., Srinivasan, R., and Williams, J. R.: Soil and water assessment tool user's manual, Version 2000, Texas Water Resources Institute: College Station, 412 p., 2002a.

Neitsch, S. L., Arnold, J. G., Kiniry, J. R., Williams, J. R., and King, K. W.: Soil and water assessment tool theoretical documentation, Version 2000, Texas Water Resources Institute: College Station, 458 p., 2002 b.

Ouma, Y. and Tateishi, R.: A water index for rapid mapping of shoreline changes of five east African rift valley lakes: An empirical analysis using Landsat TM and ETM+ data, Int. J. Remote Sens., 27, 3153-3181, 2006.

Payan, J. L., Perrin, C., Andréassian, V., and Michel, C.: How can man-made water reservoirs be accounted for in a lumped rainfall-runoff model?, Water Resour. Res., 44, W03420, doi:10.1029/2007WR005971, 2008.

Saxton, K. E. and Willey, P. H. Agricultural Wetland and Pond Hydrologic Analyses Using the SPAW Model, in: Self-Sustaining Solutions for Streams, edited by: D'Ambrosio, J. L., Wetlands, and Watersheds (12-15 September 2004, St. Paul, Minnesota, USA), St. Joseph, Michigan: ASAE, 12 September 2004, ASAE Pub \#701P0504, 16-23, 2004.

Sophocleous, M. A. and Perkins, S. P.: Methodology and application of combined watershed and ground-water models in Kansas, J. Hydrol., 236, 185-201, 2000.

Statistical Bureau of Jilin Province, Jilin Province Statistical Annual Beijing: China Statistics Press, 2001, 2000.

Wang, G. and Xia, J.: Improvement of SWAT2000 modelling to access the impact of dams and sluices on streamflow in the Huai River basin of China, Hydrolog. Process., 24, 1455-1471, 2010. 
White, M. E.: Reservoir surface area from Landsat imagery, ISPRS J. Photogramm. Eng. Remote Sens., 44, 1421-1426, 1978.

Wu, S.: A model of water body extraction based on EOS/MODIS data \& its application on flood disaster monitoring, Dissertation of Doctoral Degree. Wuhan: Huazhong University of Science and Technology, 2005.
Zhang, S. W., Zhang, Y. Z., Li, Y., and Chang, L. P.: Analysis of spatial-temporal features of land use/cover change in Northeast, Beijing: Science Press, 2006. 